\title{
Shielding of metals from the corrosion induced by water and humidity by using scratch resistant and hydrophobic silica coatings
}

\author{
M. R. Shedam ${ }^{1}$, S. S. Patil ${ }^{2}$, Rakesh M. Shedam ${ }^{3}$, Shridhar N. Mathad ${ }^{4}$ \\ ${ }^{1}$ Shikshan Maharshi D. B. Patil Research center, The New College, Kolhapur \\ 2 Vijaysinh Yadav Arts, Commerce \&5 Science college, Petvadagaon Acad. \\ ${ }^{3}$ Department of Physics, GKG college, Kolhapur 416 012, India \\ ${ }^{4}$ K.L.E.I.T., Gokul Road, Hubballi, Karnataka, India \\ E-mail: physicssiddu@gmail.com \\ mahadev.shedam@yahoo.com
}

Received: September 21, 2016

\begin{abstract}
The objective of the present paper is to demonstrate the effect of sol-gel derived scratch resistant coatings on different metal surfaces for anticorrosive applications. However, practical applications are limited by problems intrinsic to sol-gel processing or specific of coating/metal systems. Coatings aimed to improve oxidation and wet corrosion resistance have been studied the most. The results published in the literature show that sol-gel coatings may offer good protection against oxidation. More difficult is to achieve a protection against wet corrosion. An important aspect of the application of the sol-gel method for coating metallic objects is also the deposition technique.
\end{abstract}

Key words: Sol-gel process, scratch resistant, transparent silica coatings, anticorrosion.

\section{Introduction}

Hybrid sol-gel-derived polymers are attracted due to a wide range of applications. The combination of organic polymers and inorganic materials in a single phase provides promising properties to tailor electrical, optical, anticorrosive, and mechanical properties for diverse applications. Effectiveness of coating and protection of metals depends on the understanding of the process of corrosion. The steel used in metal structures is a highly processed material which is an artificially alloyed state. After refinement, metals which are unstable materials actively seek to recombine with oxygen or other elements and this process is called corrosion [1]. As the metals are constantly exposed to the corrosive environment, an effective barrier over the metal surface is needed, such as metal primers and top coat finishes. Primers and coatings have a good adhesive strength and have the ability to cover imperfection and bridge minute cavities [2-4]. The primer should be applied 
within one hour of surface preparation of the metal because of the nature of the fine micro topography substrates. Moreover, the metal will be protected against corrosion as long as sufficient coating thickness of the top coats is provided. The top coat is stable and has high resistance to abrasion and aggressive environmental conditions [5].

\section{Materials and methods}

For the preparation of scratch resistant coating the chemicals used were methyltrimethoxysilane (Sigma-Aldrich Chemie, Germany), methanol (S.D. fine Chem. Ltd, Mumbai, India) Tween 80 (Thomas Baker, Mumbai, India) and oxalic acid (Loba Chemie., India). Double distilled water was used in the preparation of the catalyst. All chemicals were used as received, without further purification. Further, 1 to 10 vol \% solution of the Tween 80 was prepared in methanol. A coating sol was prepared by keeping the molar ratio of MTMS: MeOH: $\mathrm{H}_{2} \mathrm{O}$ constant at 1: 12.04: 3.96, respectively with variation 1 to 10 vol \% Tween 80 solution with $0.1 \mathrm{M}$ of oxalic acid as a catalyst. These polymer solutions were added in the sol and deposited at room temperature on cleaned stainless steel substrates with a commercial spin coater (Chemat Technology, KW-4A Spin coater, Northridge, USA) to get thin coating of silica. The spin coating condition was $2500 \mathrm{rpm}$ for $60 \mathrm{~s}$. Further, these metal substrates were annealed at $200^{\circ} \mathrm{C}$ for $4 \mathrm{~h}$ for densification of the films and the removal of residual solvent.

\section{Results and discussion}

\subsection{Fourier transform infrared spectroscopy}

The organic modification and the hydrophobicity of the silica coatings were confirmed from Fourier Transform Infrared (FTIR) spectroscopic studies.

The coated material on metal substrate was removed and powder material exposed up to $100^{\circ} \mathrm{C}$ for one hour for removing the residual moisture. Further, coating powder was milled with potassium bromide (KBr) to form a very fine powder. This powder is compressed into a thin pellet for FTIR analysis in transmission mode, since $\mathrm{KBr}$ is transparent in the IR region.

Figure $1 \mathrm{a}$ ) and b) show the Fourier transform infrared (FTIR) spectra of the silica coating prepared without any polymer i.e. with only MTMS precursor, and with Tween 80, respectively. Several characteristic peaks were observed in the range of 4000 to $500 \mathrm{~cm}^{-1}$ indicating the presence of methyl groups in the silica coating. The absorption peek observed near $1080 \mathrm{~cm}^{-1}$, which is the characteristic peak of the Si-O-Si bond, is present in all types of coatings in the spectra [6]. The stretching and bending modes of $\mathrm{C}-\mathrm{H}$ bands were observed at 2950 and $1400 \mathrm{~cm}^{-1}$ in sample a) and b). The Si-C bonds were observed at 765 and $1265 \mathrm{~cm}^{-1}$ in both samples [7]. The new vibration near $1740 \mathrm{~cm}^{-1}$ is related to an ester group in Tween 80 (spectrum b). Actually the ester group in Tween 80 has to show vibration at $1735 \mathrm{~cm}^{-1}$ [8], but is shifted to $1740 \mathrm{~cm}-1$ probably due to the presence of Si-C groups. The FTIR spectra are suggesting that the Tween 80 is strongly associated with the silica network. The broad absorption band at $3400 \mathrm{~cm}-1$ in the spectra corresponds to the polar - $\mathrm{OH}$ bonds and the residual Si-OH groups which are the main source of hydrophilic 
character of the coating. The hydroxyl groups of the Tween 80 are coupled strongly with MTMS oligomer, leaving hydroxyl groups outside; hence the silica films are hydrophilic in nature.

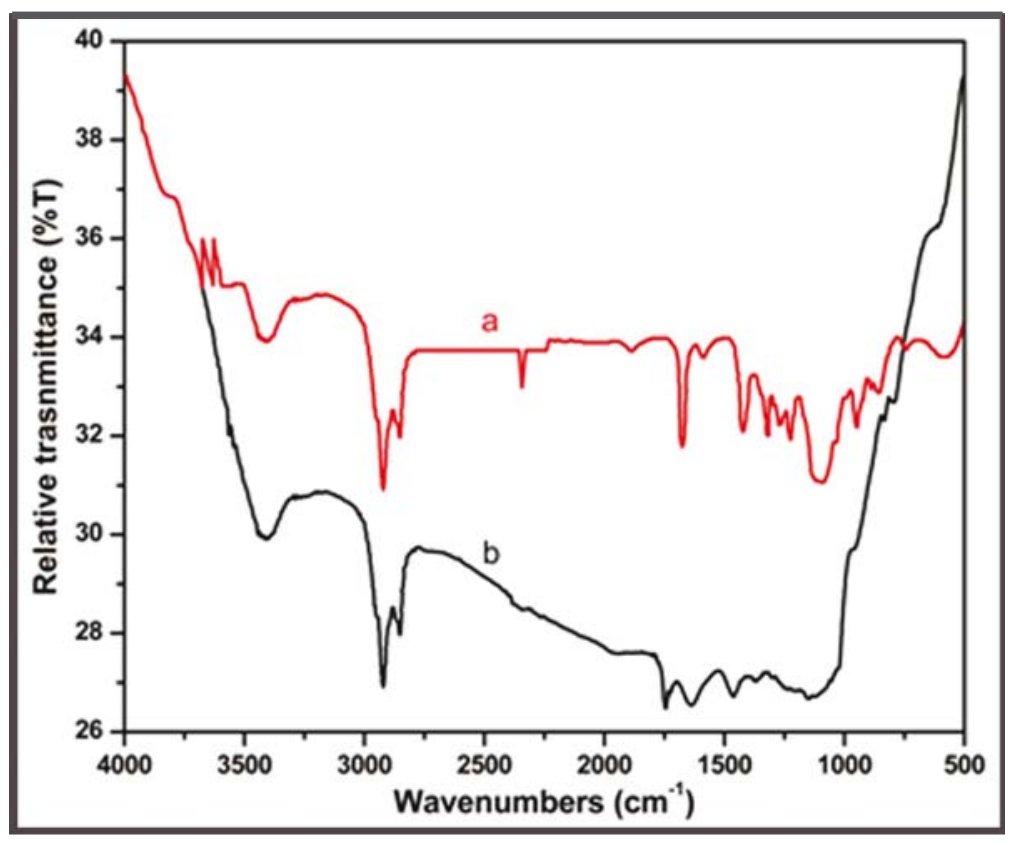

Figure 1: FTIR spectra of the silica coating on metal prepared without Tween 80 (spectrum a) and with Tween 80 (spectrum b).

\subsection{Scanning electron microscopic analysis (SEM)}

The surface morphological analysis of the scratch resistant silica film was carried out using scanning electron microscopy (JSM-6360 JEOL, Japan). SEM shows that the microstructural arrangement is inhomogeneous with grains and voids. The grain sizes of the films are around $1 \mu \mathrm{m}$ as shown in Figure $2 \mathrm{a}$ ) and b) of silica coating prepared with MTMS and Tween 80 modified silica film, respectively. The silica coated particles prepared by using only MTMS as a precursor have grains that are closely packed (more dense) and wellgrown grains randomly oriented compared to the Tween 80 sample. The silica particles of the Tween 80 modified coating are $\approx 1 \mu \mathrm{m}$ in size, sphericaly shaped and a well oriented network with ordered branching can be observed. MTMS as a precursor might increase the amount of pores is reduced owing to smaller grains. This results in individual grains coming closer to each other, therefore increasing the effective area of grain to grain contact. This results in greater densification or lesser porosity. It is observed that, when the effective concentration of dopants becomes greater (prominent) than the solid solution limit - the energy for the movement of the grain boundary - enhances [9]. The SEM micrograph of the Tween 80 modified silica coating revealed strong bonding among the silica particles. There is increase in the toughness of the coating due to this bonding which may be the reason for resistance to the surface scratches by hard objects such as the pencil scratch test. 

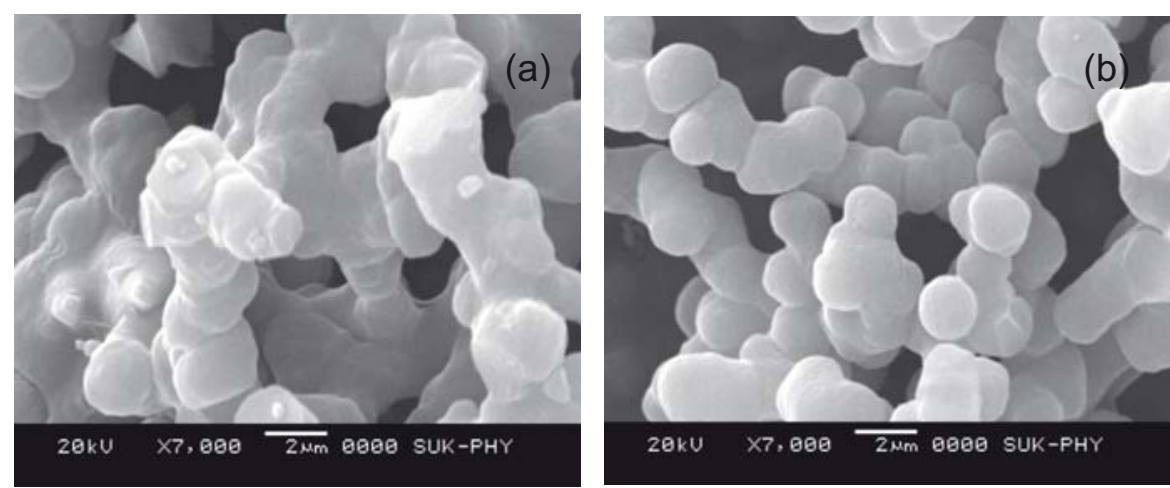

Figure 2: Scanning electron microscopic studies of the silica coatings.

\subsection{Thermal conductivity and specific heat}

The thermal conductivity was measured using a C-T meter (Teleph Company, France, accuracy $10^{-3} \mathrm{~W}^{-1} \mathrm{~m}^{-1} \mathrm{~K}^{-1}$ ) of a ring probe type which measures the thermal conductivity (K) by sandwiching the ring probe in between the powder of the coating obtained from the substrates. The rise in the temperature of the sample $(\Delta T)$ due to the supplied heating power $(0.09 \mathrm{~W})$ is given by [10]:

$$
\Delta T=\left(\frac{R I^{2}}{L}\right) \cdot\left(\frac{1}{4 \pi k}\right) \cdot\left[\ln (t)+C^{t e}\right]
$$

The thermal conductivity of the coating depends on the density of the silica network. Thermal conduction in the silica coatings occurs through solid conductivity and radiative transmission mechanisms. The decreasing nature of the variation of thermal conductivity of silica coatings prepared with Tween 80 (1-9\%) is shown in Fig.3.

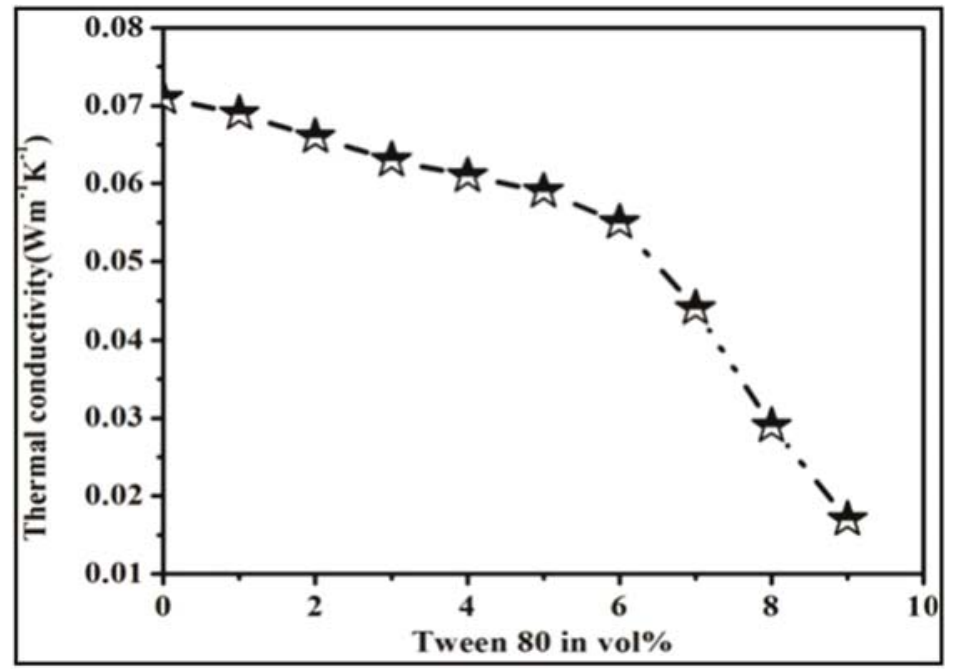

Figure 3: Variation of thermal conductivity with Tween 80 in vol (\%). 
It is observed that as wt\% of Tween 80 increased, thermal conductivity decreased from 0.071 to $0.0 .017 \mathrm{Wm}^{-1} \mathrm{~K}^{-1}$ for $0 \%$ Tween 80 and $9 \%$ Tween 80 (samples), respectively. The increase in thermal conductivity might be due to the reduction in the density of coating. It was observed that by increasing the Tween 80 content the sample transports much less thermal conductivity. The specific heat of the silica coating was found to decrease from 146 to $103 \mathrm{kJm}^{-3} \mathrm{~K}^{-1}$ with the increase in Tween 80 . Hence the more prominent the porosity, lesser is the thermally conducting solid content in the network aerogels [10]. The decrease in thermal conductivity with the increase of the PTMS/TEOS molar ratio can be explained by the fact that organic compounds have lower thermal conductivity. However, these low thermal conductive materials are extensively applicable in thermal insulation systems [10].

\subsection{Pencil scratch resistant test}

The pencil scratch resistant test of MTMS based silica coatings and the Tween 80 modified MTMS based silica coatings was done as per the ASTM D 3363 method [11]. This test uses constant pressure and variable hardness of the graphite lead as its fundamental principle. Pencils of varying hardness, starting with the hardest lead, are moved over the surface under a fixed pressure of $20.34 \mathrm{~N}(2.084 \mathrm{~kg} \pm 1 \%)$ and at a fixed angle of 45 degrees of the pencil to the surface over $20 \mathrm{~mm}$. This test was run for more than 25 cycles. No scratch on the silica film prepared with 8 vol\% of Tween 80 was recorded with the hardest pencil of $6 \mathrm{H}$. It means that Tween 80 has played the role in increasing the resistance of the silica coating to the scratches. The thickness of this film was 94 ïAnm and the surface roughness was $1298 \mathrm{~nm}$. The physical properties of the Tween 80 modified silica coatings are as shown in the Table 1.

Table 1: Physical properties of the scrath resistant silica coatings.

\begin{tabular}{cccccc}
\hline \hline \multicolumn{5}{c}{ Surface roughness of the silica coating $=37 \mathrm{~nm}$} \\
\hline $\begin{array}{c}\text { Sr. } \\
\text { No. }\end{array}$ & $\begin{array}{c}\text { Tween } 80 \\
\text { in vol\% }\end{array}$ & $\begin{array}{c}\text { Water drop } \\
\text { sliding angle } \\
\theta\left(^{\circ}\right)\end{array}$ & $\begin{array}{c}\text { Pencil Hardness } \\
\text { Grade }\end{array}$ & $\begin{array}{c}\text { Water drop } \\
\text { contact angle } \\
\theta\left(^{\circ}\right)\end{array}$ & $\begin{array}{c}\text { Thermal conductivity } \\
\left(\mathrm{Wm}^{-1} \mathrm{~K}^{-1}\right)\end{array}$ \\
\hline 1 & 0 & 41 & $6 \mathrm{~B}$ & 142 & 0.071 \\
2 & 1 & 39 & $5 \mathrm{~B}$ & 142 & 0.069 \\
3 & 2 & 28 & $\mathrm{~B}$ & 140 & 0.066 \\
4 & 3 & 27 & $\mathrm{HB}$ & 138 & 0.063 \\
5 & 4 & 26 & $\mathrm{H}$ & 137 & 0.061 \\
6 & 5 & 24 & $2 \mathrm{H}$ & 135 & 0.059 \\
7 & 6 & 19 & $3 \mathrm{H}$ & 134 & 0.055 \\
8 & 7 & 15 & $4 \mathrm{H}$ & 133 & 0.044 \\
9 & 8 & 11 & $6 \mathrm{H}$ & 131 & 0.029 \\
10 & 9 & 11 & $>6 \mathrm{H}$ & 129 & 0.017 \\
\hline \hline
\end{tabular}




\subsection{Hydrophobicity and contact angle studies}

The hydrophobic behavior of silica coating was quantified by keeping $5 \mu \mathrm{L}$ volume of the water droplet on the surface of the coating by using contact angle meter. The small value of mass of water droplet helps to ignore gravitational effects while measuring the water contact angle with the coating surface.

The contact angles photograph is shown in the Fig. 4.

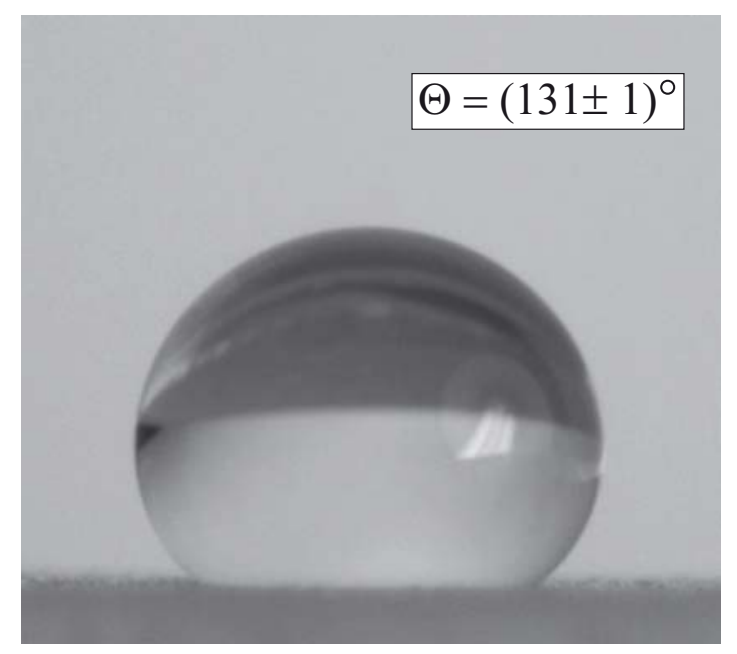

Figure 4: Water drop contact angle image on silica coated surface.

The contact angle of the water droplet of $5 \mu \mathrm{L}$ size recorded with the hydrophobic coating surface was $(131 \pm 1)^{\circ}$. Thus the high contact angle of water droplet facilitate for the prevention of metals from corrosion due to the atmospheric vapor and rain water which is crucial for the long term applications in both scientific and industrial purposes. The sliding angle is the minimum tilt of the surface from which the water droplet can be rolled off very easily. The sliding angle values of the silica coatings on the metal substrates are also given in Table 1. The sliding angle is the measure of the self cleaning ability of the silica coating on the metal surfaces. The hydrophobicity is nothing but the water repulsion ability of the coating.

\subsection{Humidity studies of the silica coatings}

The water repellent potential of hydrophobic and scratch resistant coatings gradually degrades during long term outdoor exposure and accumulation of contaminants. Therefore the stability of superhydrophobic surfaces in humid atmosphere is an important factor from application point of view. Humidity is a measure of the amount of water vapors in the air. The relative humidity $(\mathrm{H})$ is given by equation [12]:

$$
H=\frac{e}{e_{s}} \times 100
$$

where $e$ is the actual vapor pressure and $e_{s}$ is the saturated vapor pressure. In humid atmosphere, it may be mentioned that the actual vapor pressure $(e)$ is always less than the 
saturated vapor pressure $\left(e_{s}\right)$. The effect of humidity on the wetting properties of Tween 80 modified silica coating on metal substrates were carried out at relative humidity of $90 \%$ at $30^{\circ} \mathrm{C}$ over 60 days. The silica coated metal substrate prepared with 8 vol\% of Tween 80 showed the highest contact angle of $131^{\circ} \pm 1^{\circ}$. After exposing it to humid atmosphere, the contact angle decreased to $129^{\circ}$. It was observed that for all silica coatings the contact angle decreases very slightly after 60 days. The decrease in the contact angle might be due to a slight absorption of moisture from the atmosphere by the polar - $\mathrm{OH}$ bonds present in the silica coating. The samples exposed to humid atmosphere for 60 days were dried at $50{ }^{\circ} \mathrm{C}$ in an oven and the contact angle was measured for the dried samples. It was observed that the contact angle was nearly the same as before exposing the samples to the humid atmosphere. This reveals that there was no significant effect of humidity on the silica coatings. Hence the scratch resistant and hydrophobic silica coatings may have tremendous potential for anticorrosive applications.

\section{Conclusion}

There is a need towards the prevention of corrosion of the metal surfaces induced by water and humidity. The Tween 80 modified scratch resistant silica coatings showed a hydrophobic nature, hence, these coatings can be used for these purposes. The Tween 80 induces the strongest bonding between the silica particles which results in strong silica network formation that is resistant to the scratches made by the hardest pencil. It is observed that as wt\% of Tween 80 increased, thermal conductivity decreased from 0.071 to $0.0 .017 \mathrm{Wm}^{-1} \mathrm{~K}^{-1}$ for $0 \%$ Tween 80 and $9 \%$ Tween 80 (samples), respectively. The presence of the ester groups of the Tween 80 was confirmed by the FTIR analysis. The porosity of the silica network enhances the hydrophobicity of silica coatings. Hence, these scratch resistant, hydrophobic silica coatings on metal surfaces can be used as protective shields against corrosion.

\section{Acknowledgments}

The authors are grateful to the University grant commission New-Delhi, for the financial support for this work through a minor research project university grant commission 47$452 / 12(\mathrm{WRO})$.

\section{References}

[1] T. Gichuhi, W. Novelli, Waterborne Symposium Proceedings, New Orleans, pp 69-78 (2008);

The School of Polymers and High Performance Materials and Southern Society for Coatings Technology: Hattiesburg, MS,; pp 47-54 (2008).

[2] P. G. Cao, J. L. Yao, J. W. Zheng, R. A. Gu, Z. Q. Tian, Langmuir 18, 100-104 (2002). 
[3] D. Shchukin, M. Zheludkevich, K. Yasakau, S. Lamaka, M. Ferreira, H. Mohwald, Advanced Materials 18, 1672-1678 (2006).

[4] A. Nakajima, A. Fujishima, K. Hashimoto, T. Watanabe, Advanced Materials 16, 1365 (1999).

[5] N. A. Patankar, Langmuir 19, 1249 (2003).

[6] N. D. Hegde, A.V. Rao, J. Mater Sci 42, 6965-6971 (2007).

[7] R. Aelion, A. Lobel, F. Eirich, Recueil Traveux Chimiques 69, 61-75 (1950).

[8] Rong Fu, Xiumei Jin, Jinglun Liang, Weishi Zheng, Jiaqi Zhuang and Wensheng Yang, J of Materials Chemistry 21, DOI: 10.1039/c1jm11883h (2011).

[9] W. D. Kingery, H. K. Bowen, D. R. Uhlmann, Introduction to Ceramics, (2nd ed., John Wiley \& Sons, Singapore, 2004).

[10] Mahendra S. Kavale, D. B. Mahadik, V. G. Parale, P. B. Wagh, Satish C. Gupta, A. Venkateswara Rao, Applied Surface Science 258, 158-162 (2011).

[11] Standard Test Method for Pull-Off Strength of Coatings Using Portable Adhesion Testers, ASTM International, ASTM D 4541 (1998).

[12] A. V. Rao, S. S. Latthe, D. Y. Nadrgi, H. Hirashima, V. Ganeshan, Journal of Colloid and Interface Science 332, 484-490 (2009). 